\title{
Covariant space-time line elements in the Friedmann-Lemaitre-Robertson-Walker geometry
}

\section{David Escors* and Grazyna Kochan}

Fundación Miguel Servet, UPNA-IdISNA, 31008 Pamplona, Spain

*Author to whom correspondence should be addressed (descorsm@navarra.es).

\begin{abstract}
Most quantum gravity theories endow space-time with a discreet nature by space quantization on the order of Planck length $\left(\ell_{p}\right)$. This discreetness could be demonstrated by confirmation of Lorentz invariance violations (LIV) manifested at length scales proportional to $\ell_{p}$. In this paper, space-time line elements compatible with the uncertainty principle are calculated for a homogeneous, isotropic expanding Universe represented by the Friedmann-Lemaitre-Robertson-Walker solution to General Relativity (FLRW or FRW metric). To achieve this, the covariant geometric uncertainty principle (GeUP) is applied as a constraint over geodesics in FRW geometries. A generic expression for the quadratic proper space-time line element is derived, proportional to Planck length-squared and dependent on two contributions. The first is associated to the energy-time uncertainty, and the second depends on the Hubble function. The results are in agreement with space-time quantization on the expected length orders, according to quantum gravity theories and experimental constraints on LIV.
\end{abstract}

Keywords: Lorentz invariance violation, FRW metric, general relativity, quantum mechanics, uncertainty principle, quantum gravity

\section{Introduction}

General relativity (GR) is a background-independent geometric theory for gravitation in which the space-time metric is the dynamical variable $[1,2]$. The solutions to Einstein's field equations correspond to space-time metrics defined by pseudo-Riemannian metric tensors $\left(g_{\mu \nu}\right)$. In GR, particle trajectories follow geodesics in the geometries defined by such metric tensors. As the momentum/position phase space is continuous in classical GR [2], both momentum and position can be simultaneously known with absolute certainty within the geodesic trajectories. As a consequence, particle geodesics can be defined with absolute precision. This clashes with quantum mechanics, in which the momentum/position phase space is quantized. As a consequence, the measurement of 
position introduces uncertainty in momentum and viceversa. Likewise, an uncertainty relationship also exists between energy fluctuations and time intervals. These two uncertainty relationships constitute the classical Heinsenberg's uncertainty principle inequalities of quantum mechanics $[3,4]$ :

$$
|\Delta p \Delta x| \geq \frac{\hbar}{2}, \quad|\Delta E \Delta t| \geq \frac{\hbar}{2} .
$$

where $\Delta p$ and $\Delta x$ represent the change in the magnitude of momentum and position, respectively; $\Delta E$ and $\Delta t$ represent the change in magnitude of energy and time, respectively; $\hbar$ is the reduced Planck constant.

These inequalities inherently reflect the quantization of the momentum-position phase space in units of $\hbar$. From these inequalities it is deduced that the subjacent space-time geometry must also be fundamentally discrete. Attempts to quantize the space-time geometry by several methods have led to quantum gravity theories such as Loop Quantum Gravity, or LQG $[5,6]$. From the process of space quantization, current quantum gravity theories rely on a minimum space-time length for their formulation, which is proportional to Planck length $\left(\ell_{p}\right)$. For example, the quanta of area and volume operators in LQG are proportional to $\ell_{p}^{2}$ and $\ell_{p}^{3}$, respectively [7-9]. Likewise, other quantum gravity theories such as string theory also introduce $\ell_{p}$ as a fundamental length element for particles [10-12]. Most quantum gravity theories predict phenomena such as in vacuo dispersion of photons and neutrinos, and deviations of photon polarization over astronomical distances caused by Lorentz invariance violations (LIV) [13-18]. These phenomena are predicted to arise if indeed the space-time is discreet. LIV confirmation could constitute a major step forward to proving quantum space-time discreetness.

The process of spatial quantization or the establishment of a fundamental length associated to particles alter the classical uncertainty principle, leading to formulations such as the Generalized Uncertainty Principle, or GUP [19-21]:

$$
|\Delta p \Delta x| \geq \frac{\hbar}{2}+\frac{\hbar c^{2}}{m^{2} A^{2} \delta s^{2}} \Delta p^{2} .
$$

This expression includes a correction dependent on the particle mass, $m$, the proper acceleration, $A$, the speed of light, $c$, and the quadratic form of the space-time length element $\delta s^{2}$.

As the space-time metric in GR is shaped by energy-momentum densities through the energy-momentum tensor, vacuum energy and momentum fluctuations from the uncertainty principle should perturb the space-time $[19,22]$. Indeed, very early on it was assumed that in the context of a quantum description of gravity, quantum fluctuations caused by Heisenberg's principle play a major role [23]. In semi-classical descriptions of quantum gravity, a putative metric tensor operator, $\hat{g}_{\mu \nu}$, is decomposed in the classical pseudo-Riemannian metric tensor, $g_{\mu \nu}$, and a fluctuating tensor operator of quantum origin, $\delta \hat{g}_{\mu \nu}$, which introduces a differential perturbation [24]: 


$$
\hat{g}_{\mu \nu}=g_{\mu \nu}+\delta \hat{g}_{\mu \nu} .
$$

The indices, denoted by Greek letters take on the values 0, 1, 2 and 3, defining the temporal and spatial components in standard relativistic tensor notation. The expectation value of the perturbation is then identified with a quantum-associated classical tensor $T_{\mu \nu}$ :

$$
\left\langle\delta \hat{g}_{\mu \nu}\right\rangle \equiv T_{\mu \nu}
$$

A more direct relationship between the uncertainty principle and alterations to the metric can be formulated with quantum mechanics commutators, used in string theories and quantum topology [19]. A momentum-position commutator is thus associated to the Minkowski metric tensor, that is then generalized to curved space-time through a pseudo-Riemannian metric tensor:

$$
\left[P^{\mu}, X^{\nu}\right]=-i \hbar \eta^{\mu \nu}, \quad\left[P^{\mu}, X^{\nu}\right]=-i \hbar g^{\mu \nu} .
$$

where $P^{\mu}, X^{v}$ stand for the components of momentum and position 4-vector operators. $\eta^{\mu \nu}, g^{\mu \nu}$ represent the contravariant Minkowski and pseudo-Riemannian metric tensors, respectively.

Independently of the specific model for quantum gravity, uncertainty fluctuations introduce a perturbation in the metric that is unrelated to classical gravitation, but can be otherwise related to a minimal length for the space-time line element [19,22,25-27]. However, the use of a fixed length for the line element clashes with classical relativity. Indeed, the LQG minimal length could be considered a "free parameter" [28], complying with Lorentz co-variance [21]. This has led to corrections to the canonical GUP momentum-position commutator for Minkowski space as shown in [21]:

$$
\left[P^{\mu}, X^{\nu}\right]=-i \hbar \eta^{\mu \nu}-i \hbar \mathrm{A} \eta^{\mu \nu}-i \hbar \mathrm{B}\left(P^{\mu}, P^{v}\right) .
$$

with $A$ and $B$ being functions of momentum.

The classical uncertainty principle can also be reformulated as a relativistic covariant form in terms of the proper space-time line element $\left(\tau^{2}\right)$ and Planck length, $\ell_{p}$ [29]. This reformulation allows its application as a mathematical constraint over GR geodesics without an explicit quantization of space-time. The differential quadratic proper spacetime line element is then defined as a function of Planck length through a geodesicderived scalar, $\mathrm{G}_{\text {geo: }}$ :

$$
\left|G_{\text {geo }} d \tau^{2}\right| \geq(1+\gamma) \ell_{p}^{2}
$$

where $d \tau$ is the proper space-time line element, and the gamma factor $\gamma$ and Geodesic scalar are defined:

$$
\begin{gathered}
\gamma=\frac{d t}{d \tau} \equiv \frac{E}{m}, \\
G_{\mathrm{geo}} \equiv 2 G m\left|U_{0} \Gamma_{\alpha \beta}^{0} U^{\alpha} U^{\beta}\right|+2 G m\left|U_{1} \Gamma_{\alpha \beta}^{1} U^{\alpha} U^{\beta}\right| .
\end{gathered}
$$


where $E$ corresponds to the total energy of the particle; $m$ corresponds to its massenergy in units of $c$ set to $1 ; \Gamma_{\alpha \beta}^{\mu}$ corresponds to Christoffel symbols calculated from the pseudo-Riemannian metric tensor; $\mathrm{G}$ is the universal gravitational constant; $U_{\alpha}, U^{\alpha}$ are covariant and contravariant components of proper velocity. The indices, denoted by Greek letters take on the values 0, 1, 2 and 3, defining the temporal and spatial components in standard relativistic tensor notation.

This formulation sets a length limit for the quadratic proper space-time line element which is proportional to $\ell_{p}^{2}$, and defined by the metric tensor. This covariant form of the classical uncertainty principle can be applied to any GR solution. For example, a welldefined minimum space-time line element in a Schwarzschild black hole singularity at radial position $\mathrm{R}=0$ was calculated in [29]:

$$
\left|d \tau^{2}\right| \geq \frac{2 M}{m} \ell_{p}^{2}
$$

Where $M$ is the mass of the black hole generating the gravitational field, and $m$ is the mass of the particle.

The exact solution to Einstein's field equations for a homogeneous, isometric Universe that expands following Hubble's law corresponds to the FRW (or FLRW) metric [30,31]. This solution represents a first approximation to the standard model of Cosmology. In units of $c$ set to 1 with a $(-+++)$ Lorentzian metric signature, its line element is represented:

$$
d \tau^{2}=-d t^{2}+a^{2} \frac{d R^{2}}{1-K R^{2}}+a^{2} R^{2} d \theta^{2}+a^{2} R^{2} \sin ^{2} \theta d \varphi^{2} .
$$

Where $t$ corresponds to the temporal coordinate and $R, \theta$ and $\varphi$ to dimensionless comoving polar coordinates. The time-dependent universal scale factor, $a$, provides dimensions of length to the co-moving coordinates, and determines the physical size of the Universe; The curvature constant, $K$, takes on values of 1,0 or -1 , depending on the model of the expanding Universe; closed-spherical, flat or open-hyperbolic, respectively. Although the Universe presents a very small positive curvature, for practical purposes it can be discarded. Indeed current measurements of cosmological parameters are in agreement with a spatially-flat cosmology [32]. Hence, $K$ set to zero could be justified to describe the current state of the Universe.

In principle, it could be considered that the lengths for the space-time line element in the FRW metric depend on two factors: first, energy-time quantum fluctuations, and second, the expansion rate of the Universe as defined by Hubble's function, $H$, calculated from the scale factor:

$$
H=\frac{\dot{a}}{a} \quad ; \quad \dot{a} \equiv \frac{d a}{d t}
$$

In this paper, GeUP was applied to the FRW solution to define length restrictions on the space-time line element in the context of a covariant formulation which conserves Lorentz invariance. The results from the calculations are then discussed in the context 
of the predicted length elements in quantum gravity theories, and the experimental restrictions upon Lorentz invariance violations calculated from experimental observations.

\section{Space-time line element for a particle at rest in the FRW metric.}

Unless otherwise stated, the equations will be expressed in units of $c$ set to 1 . Standard relativistic tensor notation will used, with temporal and polar spatial coordinates designated as: $\left(X^{0}, t\right) ;\left(X^{1}, R\right) ;\left(X^{2}, \theta\right) ;\left(X^{3}, \varphi\right)$. The most general FRW solution is given by equation (10). A particle at rest implies the following statements on proper velocities:

$$
U^{1}=U^{2}=U^{3}=0 \quad, \quad U^{0} U_{0}=-1 .
$$

To apply GeUP from inequality (7), the geodesic scalar is first calculated:

$$
G_{\text {geo }}=2 G m\left|U_{0} \Gamma_{00}^{0} U^{0} U^{0}\right| \text {. }
$$

The only Christoffel connector that participates in the calculation is $\Gamma_{00}^{0}$, which is zero in the FRW metric. Hence, the geodesic scalar is also 0 , leading to a contradiction in inequality (7) unless Plank length is considered zero in the non-quantum limit:

$$
0 \geq(1+\gamma) \ell_{p}^{2}
$$

Therefore, the classical FRW metric is incompatible with the uncertainty principle unless a $t$-dependent differential perturbation function, $\varepsilon$, is introduced in the $g_{00}$ component of the metric, following a similar approach by semi-classical quantum gravity and developed for Minkowski space in [29]:

$$
g_{00}=-1-\varepsilon(t) .
$$

The introduction of this differential perturbation in the FRW metric leads to a compatible solution with the uncertainty principle without modifying the overall solution:

$$
d \tau^{2}=-(1+\varepsilon) d t^{2}+a^{2} \frac{d R^{2}}{1-K R^{2}}+a^{2} R^{2} d \theta^{2}+a^{2} R^{2} \sin ^{2} \theta d \varphi^{2} .
$$

The term $(1+\gamma)$ in inequality (7) takes a value of 2 for a particle at rest. After the calculation of the geodesic scalar and Christoffel connector as described in [29], one obtains inequality (7) as:

$$
\left|d t^{2}\right| \geq \frac{2 \ell_{p}^{2}}{\left|G P^{0} \dot{\varepsilon}\right|}, \quad \dot{\varepsilon} \equiv \frac{d \varepsilon}{d t} .
$$

This expression can be re-written according to the classical energy-time uncertainty:

$$
\left|P^{0} d \varepsilon d t\right| \geq \hbar \quad, \quad P^{0} d \varepsilon \equiv d E .
$$


where $P^{0} d \varepsilon$ corresponds to energy variations of the particle in the geodesic in units of $c$ set to 1 . Therefore, $P^{0} \dot{\varepsilon}$ corresponds to time-dependent energy fluctuations of the particle in the geodesic:

$$
P^{0} \dot{\varepsilon}=\frac{P^{0} d \varepsilon}{d t}=\frac{d E}{d t} \equiv \dot{E} .
$$

where dots over variables represent derivatives of the indicated variables with the time coordinate. And after recovering $c$ in inequality (17), one ends in units of time with:

$$
\left|d t^{2}\right| \geq \frac{2 c^{3} \ell_{p}^{2}}{G|\dot{E}|} \sim \frac{1}{|\dot{E}|} O\left(10^{-34}\right) .
$$

2. Generic solution for the FRW space-time line element for a moving particle in the $\mathbf{R}$ coordinate

The FRW symmetry allows simplification of calculations by considering geodesics moving in the R coordinate without displacements in the angular coordinates. In this condition only the components of proper velocities for $t$ and $R$ coordinates will contribute to the calculation of the Geodesic scalar:

$$
G_{\mathrm{geo}} \equiv 2 G\left|U_{0} \Gamma_{\alpha \beta}^{0} U^{\alpha} U^{\beta}\right|+2 G\left|U_{1} \Gamma_{\alpha \beta}^{1} U^{\alpha} U^{\beta}\right| .
$$

Expanding this expression one gets:

$$
\begin{aligned}
G_{\text {geo }} \equiv 2 G\left|U_{0} \Gamma_{00}^{0} U^{0} U^{0}+2 U_{0} \Gamma_{01}^{0} U^{0} U^{1}+U_{0} \Gamma_{11}^{0} U^{1} U^{1}\right| \\
+2 G\left|U_{1} \Gamma_{00}^{1} U^{0} U^{0}+2 U_{1} \Gamma_{10}^{1} U^{1} U^{0}+U_{1} \Gamma_{11}^{1} U^{1} U^{1}\right| .
\end{aligned}
$$

The contributing Christoffel connectors are:

$$
\begin{array}{r}
\Gamma_{00}^{0}=\frac{\dot{\varepsilon}}{2(1+\varepsilon)} \quad ; \quad \Gamma_{01}^{0}=\Gamma_{00}^{1}=0 \quad ; \quad \Gamma_{11}^{1}=\frac{K R}{\left(1-K R^{2}\right)} \\
\Gamma_{11}^{0}=\frac{a \dot{a}}{(1+\varepsilon)\left(1-K R^{2}\right)}+\frac{a^{2} K R \dot{R}}{(1+\varepsilon)\left(1-K R^{2}\right)^{2}} \quad ; \quad \Gamma_{10}^{1}=H+\frac{K R \dot{R}}{1-K R^{2}} .
\end{array}
$$

where dots over the $R$ coordinate, the scale factor and the metric perturbation indicate differentiation by time coordinate. Calculating the geodesic scalar with the non-zero terms one gets:

$$
\begin{aligned}
G_{\text {geo }} & \equiv 2 G m\left|U_{0} \Gamma_{00}^{0} U^{0} U^{0}+U_{0} \Gamma_{11}^{0} U^{1} U^{1}\right| \\
& +2 G m\left|2 U_{1} \Gamma_{10}^{1} U^{1} U^{0}+U_{1} \Gamma_{11}^{1} U^{1} U^{1}\right| .
\end{aligned}
$$

And introducing the explicit terms for the Christoffel connectors one gets: 


$$
\begin{aligned}
G_{\text {geo }} \equiv 2 G m \mid & U_{0} \frac{\dot{\varepsilon}}{2(1+\varepsilon)} U^{0} U^{0} \\
& +U_{0} U^{1} U^{1}\left(\frac{a \dot{a}}{(1+\varepsilon)\left(1-K R^{2}\right)}+\frac{a^{2} K R \dot{R}}{(1+\varepsilon)\left(1-K R^{2}\right)^{2}}\right) \mid \\
& +2 G m \mid 2 U_{1} U^{1} U^{0}\left(H+\frac{K R \dot{R}}{1-K R^{2}}\right) \\
& +U_{1} U^{1} U^{1}\left(\frac{K R}{\left(1-K R^{2}\right)}\right) \mid
\end{aligned}
$$

The expression can be simplified as a function of contravariant $U^{0}$ and the use of some equalities:

$$
\begin{aligned}
& U^{1}=U_{1} g^{11}+U_{0} g^{01}=U_{1} \frac{1-K R^{2}}{a^{2}}, \\
& U_{0}=U^{0} g_{00}=-(1+\varepsilon) U^{0} \\
& U_{0} U^{0}+U_{1} U^{1}=-1 .
\end{aligned}
$$

which leaves the geodesic scalar:

$$
\begin{aligned}
G_{\text {geo }} \equiv 2 G m \mid & \left(-1-U_{1} U^{1}\right) \frac{\dot{\varepsilon}}{2(1+\varepsilon)} U^{0}-U^{1} U_{1} U^{0}\left(H+\frac{K R \dot{R}}{1-K R^{2}}\right) \mid \\
& +2 G m \mid 2 U_{1} U^{1} U^{0}\left(H+\frac{K R \dot{R}}{1-K R^{2}}\right) \\
& +U_{1} U^{1} U^{1}\left(\frac{K R}{1-K R^{2}}\right) \mid .
\end{aligned}
$$

and it is equivalent to:

$$
\begin{gathered}
G_{\text {geo }} \equiv \mid \frac{2 G m\left(1+U_{1} U^{1}\right) \dot{\varepsilon}}{2(1+\varepsilon)} U^{0} \quad+6 G m U^{1} U_{1} U^{0}\left(H+\frac{K R \dot{R}}{1-K R^{2}}\right) \\
+2 G m U_{1} U^{1} U^{1}\left(\frac{K R}{1-K R^{2}}\right) \mid .
\end{gathered}
$$

To simplify the expression, a curvature-associated factor, F, is defined:

$$
F \equiv \frac{K R}{1-K R^{2}}
$$

and the Geodesic scalar takes the final form of:

$$
G_{\text {geo }}=\left|\frac{G m\left(1+U_{1} U^{1}\right) \dot{\varepsilon}}{1+\varepsilon} U^{0}+6 G m U^{1} U_{1} U^{0}(H+F \dot{R})+2 G m U_{1} U^{1} U^{1} F\right| .
$$

Incorporating this term into inequality (7): 


$$
\left|d \tau^{2}\right| \geq \frac{(1+\gamma) \ell_{p}^{2}}{G\left|\frac{\left(1+U_{1} U^{1}\right) \dot{\varepsilon}}{1+\varepsilon} P^{0}+6 U^{1} U_{1} P^{0}(H+F \dot{R})+2 U_{1} U^{1} P^{1} F\right|} .
$$

A flat space-time is an accurate model for current cosmological models, which corresponds to $F$ set to zero. Additionally, the epsilon correction can be ignored in $1+$ $\varepsilon$ :

$$
\left|d \tau^{2}\right| \geq \frac{(1+\gamma) \ell_{p}^{2}}{G P^{0}\left|6 U^{1} U_{1} H-U^{0} U_{o} \dot{\varepsilon}\right|} .
$$

The quadratic length for the FRW space-time line element thus depends on two factors. The first one, a function derived from the energy-time uncertainty $\left(E_{u n}\right)$, and the second one dependent on the expansion rate of the universe $\left(H_{e x}\right)$ :

$$
\begin{aligned}
& E_{u n} \equiv U^{0} U_{o} \dot{\varepsilon}, \quad H_{e x} \equiv 6 U^{1} U_{1} H \\
& \left|d \tau^{2}\right| \geq(1+\gamma) \frac{\ell_{p}^{2}}{G P^{0}\left|H_{e x}-E_{u n}\right|} .
\end{aligned}
$$

\section{Specific solutions for the FRW space-time line element for a moving particle in the $\mathrm{R}$ coordinate}

One can consider several scenarios depending on which of the functions is dominant in the denominator of inequality (33). If $E_{u n}$ is several orders of magnitude larger than $H_{\text {ex }}$, then the expression simplifies as:

$$
\left|d \tau^{2}\right| \geq \frac{(1+\gamma) \ell_{p}^{2}}{G P^{0}\left|-U^{0} U_{o} \dot{\varepsilon}\right|} .
$$

Considering the following equalities for a particle at non-relativistic velocities in units of c set to 1:

$$
(1+\gamma)=2 \quad, \quad P^{0} \dot{\varepsilon} \equiv \dot{E} \quad ; \quad U^{0} U_{o} \equiv \frac{E^{2}}{m^{2}} .
$$

And after incorporating these equalities into inequality (34), it takes the form:

$$
\left|d \tau^{2}\right| \geq \frac{2 m^{2} \ell_{p}^{2}}{G\left|E^{2} \dot{E}\right|} .
$$

In units of $c$ set to 1 , the mass term, $m$, is of the same order of energy than the total energy of particles moving with non-relativistic velocities. This will lead to further simplifications, and after re-introducing $c$ one gets:

$$
\left|d \tau^{2}\right| \geq \frac{2 c^{5} \ell_{p}^{2}}{G|\dot{E}|}
$$


The minimum allowed length for the space-time line element in the FRW metric is inverse to the rate of change of energy fluctuations of the particle in the geodesic. The quadratic space-time line element is then :

$$
\left|d \tau^{2}\right| \sim \frac{1}{|\dot{E}|} O\left(10^{-18}\right) .
$$

This expression is the same as inequality (20) but in units of length.

Quantum energy fluctuations can be neglected, for example, in large objects moving at non-relativistic velocities, then the following equalities are fulfilled:

$$
|\dot{\varepsilon}| \sim 0 \quad ;(1+\gamma)=2 .
$$

This condition makes the $H_{e x}$ term dominant in inequality (33):

$$
\left|d \tau^{2}\right| \geq \frac{\ell_{p}^{2}}{3 G P^{0}\left|U^{1} U_{1} H\right|} .
$$

To simplify this expression, the mass, $m$, can be incorporated into one of the radial proper velocities to convert it to proper momentum:

$$
\left|d \tau^{2}\right| \geq \frac{m \ell_{p}^{2}}{3 G P^{0}\left|P^{1} U_{1} H\right|} .
$$

In units of $c$ set to 1 , the mass-energy term, $m$, is of the same order than $P^{0}$ leading to their simplification:

$$
\left|d \tau^{2}\right| \geq \frac{\ell_{p}^{2}}{3 G\left|P^{1} U_{1} H\right|}
$$

And recovering $c$ into the equation:

$$
\left|d \tau^{2}\right| \geq \frac{c^{5} \ell_{p}^{2}}{3 G\left|P^{1} U_{1} H\right|} \sim \frac{1}{H E_{k}} O\left(10^{-18}\right) .
$$

$P^{1} U_{1}$ is a term proportional to proper kinetic energy. The minimum quadratic length for the space-time line element, which is of the order of $10^{-18}$, is modified by the kinetic energy of the particle and the expansion rate of the Universe.

\section{Discussion}

In this paper, a covariant form of the uncertainty principle [29] is applied as a constraint over FRW geometries to obtain expressions for the quadratic space-time line elements in a homogeneous, isometric Universe which expands according to Hubble's law. The imposition of GeUP introduces a quantization condition of the momentum-position phase space on the classical GR geodesic. This mathematical process implicitly implies a quantization of the subjacent space-time, leading to expressions for the quadratic space-time line element which are proportional to Planck length squared. This is in 
agreement with most quantum gravity theories. It has to be remarked that this mathematical constraint does not truly constitute a quantum gravity theory per se. It relies on specific solutions to classical GR equations, without coupling an external mass field, unlike semi-classical formulations of quantum gravity [22]. Explicit quantization of space-time is not introduced as well. However, the results from this mathematical constraint over classical GR solutions may approximate to the theoretical limits of lengths for the space-time line element in specific GR solutions compatible with quantum gravity phenomena.

The experimental determination of the length scales of space-time quantization is critical to set up proper mathematical constraints for quantum gravity, and discard incompatible scenarios [13]. For example, it would help on deciding the correct lattice quantization of space-time in LQG, and its properties regarding the time problem and the need for privileged reference frames [5,25,33,34]. Most current quantum gravity theories such as LQG and string theory $[6,10]$ predict that LIV occurs by the discrete nature of space-time. Hence, the experimental testing of LIV could not only demonstrate space-time quantization, but also the scales of lengths and energies in which quantum gravity acts [18].

Putative upper limits to the constraints to LIV have been experimentally estimated by several means [14-17]. LIV is predicted by quantum gravity theories to affect energy and helicity-dependent photon propagation velocities, which could be measured when accumulated over astronomical distances. Thus, by measuring deviations of photons from gamma ray bursts (GRB 041219A) an upper limit of $1.110^{-14}$ on the vacuum birefringence effect was estimated [16]. This constraint on Lorentz invariant violation would translate into "spatial volume units" of the order of $10^{-42}$ or less. Some recent studies are providing convincing LIV violations experimentally at different energy orders, while other studies stablish very stringent constraints for LIV, or even fail to detect it $[13,15,16,35,36]$.

The constraints to the space-time line elements calculated in this paper are strictly obtained using a relativistic covariant formulation of the uncertainty principle in momentum and position 4-vectors. To obtain such constraints, GeUP [29] was applied to the FRW metric as an approximation to current cosmological models [31]. More specifically, a flat geometry condition was applied to the solution as it agrees with current observations [32]. As expected, the length for the space-time line element depends on terms derived from the energy-time uncertainty, and from the Hubble function. This last term is only predominant if energy quantum fluctuations can be ignored, for example in large astronomical objects.

The calculated lengths in this paper are compatible with those from other quantum gravity theories, and with current constraints estimated for LIV. For example, the quanta of area and volume in LQG are proportional to $\ell_{p}^{2}$ and $\ell_{p}^{3} \quad[7,8,12]$, and $\ell_{p}$ constitutes a natural unit in string theories and doubly special relativity $[10,11,37,38]$. These fundamental volume blocks would be in agreement with the experimental constraints found for LIV. If confirmed, the discreet nature of space-time would have been proven. 
Such small quanta of space-time arise also in this paper. It has to be remarked that all expressions for the quadratic line elements obtained in this paper are proportional to

$\ell_{p}^{2}$, indicating that lengths of the order of $\ell_{p}$ constitute the "building blocks" of the space-time. Nevertheless, the scales in which space-time quantisation could be experimentally tested could be much wider. As shown in this paper, the calculated quanta of volume would be on the range of $10^{-27} \mathrm{~m}^{3}$ but further modified according to corrections depending on quantum energy fluctuations and Hubble's function.

\section{Author Contributions}

Conceptualization, D.E. and G.K.; methodology, D.E; resources, D.E. and G.K. All authors have read and agreed to the published version of the manuscript.

\section{Funding}

D.E. is funded by a Miguel Servet Fellowship (ISCIII, Spain, Ref CP12/03114).

\section{Conflicts of Interest}

The authors declare no conflict of interest.

1. Einstein, A. Grundlage der allgemeinen Relativitätstheorie. Annalen der Physik 1916, 49, 769-822.

2. Ashtekar, A.; Lewandoski, J. Background Independent Quantum Gravity: A Status Report. Class.Quant.Grav. 2004, 21, R53-R152, doi:10.1088/02649381/21/15/R01.

3. Ozawa, M. Heisenberg's original derivation of the uncertainty principle and its universally valid reformulations. Curr. Sci 2015, 109, 2006-2016.

4. Werner, R.F.; Farrely, T. Uncertainty from Heisenberg to today. Found. Phys. 2019, 49, 460-491, doi:10.1007/s10701-019-00265-z.

5. Rovelli, C. Loop Quantum Gravity. Living Rev. Rel. 2008, 11, 1-69, doi:10.12942/Irr-2008-5.

6. Aharony, O.; Gubser, S.S.; Maldacena, J.; Ooguri, H.; Oz, Y. Large N field theories, string theory and gravity. Phys. Rep. 2000, 323, 183-386, doi:10.1016/S03701573(99)00083-6.

7. Helesfai, G.; Bene, G. A numerical study of spectral properties of the area operator y loop quatum gravity. arXiv:gr-qc/0306124 2003.

8. Ashtekar, A.; Lewandoski, J. Quantum theory of geometry. I: Area operators. Class. Quant. Grav. 1997, 14, A55-A82, doi: 10.1088/0264-9381/14/1A/006.

9. Brunnemann, J.; Rideout, D. Properties of the Volume operators in Loop Quantum Gravity I: Results. Class. Quant. Grav. 2008, 25, 065001, doi:10.1088/0264-9381/25/6/065001.

10. Gross, D.J.; Mende, P.F. String theory beyond the Planck scale. Nucl. Phys. B. 1988, 303, 407-454. 
11. Magueijo, J.; Smolin, L. String theories with deformed energy momentum relations, and a possible non-tachyonic bosonic string. Phys. Rev. D 2005, 71, 026010, doi:10.1103/PhysRevD.71.026010.

12. Casares, P.A.M. A review on Loop Quantum Gravity. arXiv:1808.01252 2018.

13. Burderi, L.; Sanna, A.; Di Salvo, T.; Amati, L.; Amelino-Camelia, G.; Branchesi, M.; Capozziello, S.; Coccia, E.; Colpi, M.; Costa, E., et al. GrailQuest: hunting for Atoms of Space and Time hidden in the wrinkle of Space-Time. arXiv:1911.02154v2 2020.

14. Ellis, J.; Konoplich, R.; Mavromatos, N.E.; Nguyen, L.; Sakharov, A.S.; SarkisyanGrinbaum, E.K. Robust constraint on Lorentz violation using Fermi-LAT gammaray burst data. Phys. Rev. D 2019, 99, 083009, doi:10.1103/PhysRevD.99.083009.

15. Wei, J.J.; Wu, X.F. A Further Test of Lorentz Violation from the Rest-frame Spectral Lags of Gamma-Ray Bursts. ApJ 2017, 851, 127, doi:10.3847/15384357/aa9d8d.

16. Laurent, P.; Gotz, D.; Binetruy, P.; Covino, S.; Fernandez-Soto, A. Constraints on Lorentz Invariance Violation using INTEGRAL/IBIS observations of GRB041219A. Phys. Rev. D 2011, 83, 121301(R).

17. Maccione, L.; Liberati, S.; Celotti, A.; G., K.J.; Ubertini, P. Gamma-ray polarization constraints on Planck scale violations of special relativity. Phys. Rev. D 2008, 78, 103003.

18. Jacobson, T.; Liberati, S.; Mattingly, D. Lorentz violation at high energy: concepts, phenomena and astrophysical constraints. Annals Phys. 2006, 321, 150-196.

19. Capozziello, S.; Lambiase, G.; Scarpetta, G. Generalized uncertainty principle from quantum geometry. Int. J. Theor. Phys. 2000, 39, 15-22.

20. Das, S.; Vanegas, E.C. Phenomenological implications of the generalized uncertainty principle. Can. J. Phys. 2009, 87, 233-240, doi:10.1139/P08-105.

21. Todorinov, V.; Bosso, P.; Das, S. Relativistic generalized uncertainty principle. Annals Phys. 2019, 405, 92-100, doi:10.1016/j.aop.2019.03.014.

22. Haghani, Z.; Harko, T. Effects of quantum metric fluctuations on the cosmological evolution in Friedmann-Lemaitre-Robertson-Walker geometries. Physics 2021, 3, 689-714, doi:10.3390/physics3030042.

23. Wheeler, J.A. On the nature of quantum geometrodynamics. Ann. Phys 1957, 2, 604.

24. Dzhunushaliev, V.; Folomeev, V.; Kleihaus, B.; Kunz, J. Modified gravity from the quantum part of the metric. Eur. Phys. J. C 2014, 74, 1-4.

25. Hossenfelder, S. Minimal length scale scenarios for quantum gravity. Living Rev. Rel. 2013, 16, doi:https://doi.org/10.12942/lrr-2013-2.

26. Hamber, H.W. Quantum gravitation; Springer: 2009.

27. Kempf, A.; Mangano, G.; Mann, R.B. Hilbert space representation of the minimal length uncertainty relation. Phys. Rev. $D$ 1995, 52, 1108, doi: 10.1103/PhysRevD.52.1108.

28. Dzierzak, P.; Jezierski, J.; Malkiewicz, P.; Piechocki, W. The minimum length problem of loop quantum cosmology. Acta Phys. Pol. B 2010, 41, 717-726.

29. Escors, D.; Kochan, G. Constraints on general relativity geodesics by a covariant geometric uncertainty principle. Physics 2021, 3, 790-798, doi:10.3390/physics3030049. 
30. Lachieze-Rey, M.; Luminet, J.P. Cosmic tolopogy. Phys. Rep. 1995, 254, 135-214, doi:10.1016/0370-1573(94)00085-H.

31. Mueller, T.; Grave, F. Catalogue of Spacetimes. arXiv:0904.4184v32010.

32. Aghanim, N.; Akrami, Y.; Ashdown, M.; Aumont, J.; Baccigalupi, C.; Ballardini, M.; Banday, A.J.; Barreiro, R.B.; Bartolo, N.; Basak, S., et al. Planck 2018 results: VI. Cosmological parameters. Astron. Astrophys. 2020, 641, A6, doi:10.1051/00046361/201833910.

33. Anderson, E. The problem of time in quantum gravity. arXiv:1009.2157v32012.

34. Vucetich, H. Testing Loretnz invariance violation in quantum gravity theories. arXiv:gr-qc/0502093v1 2005.

35. Amelino-Camelia, G.; D’Amico, N.; Rosati, G.; Loret, N. In-vacuo-dispersion features for GRB neutrinos and photons. Nat. Astron. 2017, 1, 0139, doi:10.1038/s41550-017-0139.

36. Xu, H.; Ma, B.Q. Light speed variation from gamma-ray bursts. Astropart. Phys. 2016, 82, 72, doi: $\quad$ 10.1016/j.astropartphys.2016.05.008.

37. Amelino-Camelia, G. Doubly-special relativity: facts, myths and some key open issues. Symmetry 2010, 2, 230.

38. Susskind, L.; Uglum, J. String physics and black holes. ucl.Phys.Proc.Suppl 1996, 45BC, 115-134, doi:10.1016/0920-5632(95)00630-3. 